OAI-PMH: http://www.indteca.com/ojs/index.php/Revista Scientific/oai

Artículo Original / Original Article

\title{
Juegos Educativos Implementados por el Docente como Estrategia para el Conocimiento Matemático de los Niños
}

Autor: Carlos Reinaldo Patiño Espinal Universidad Fermín Toro, UFT carlosreypati58@gmail.com Lara, Venezuela https://orcid.org/0000-0003-3843-0850

Resumen

El presente artículo representa un aporte significativo para la enseñanza de la disciplina matemática. El mismo se titula juegos educativos implementados por el docente como estrategia para el conocimiento matemático. Tuvo como objetivo describir los juegos educativos implementados por el docente como estrategia para el conocimiento matemático en los niños de la EIB "María Clementina de Bonilla" del municipio Peña estado Yaracuy. Metodológicamente, se fundamentó en un estudio de carácter descriptivo apoyado en una investigación de campo. La población la conformó quince (15) docentes los cuales constituyeron la muestra tratándose de una muestra censal. Fue aplicado un cuestionario con escala tipo Likert. Para los resultados obtenidos se utilizó la estadística descriptiva de frecuencia y porcentaje representados en cuadros. Dentro de las conclusiones se destacó baja tendencia de los docentes en implementar los juegos educativos como estrategia para el conocimiento matemático. Considerando poca importancia a los objetivos del juego como elemento que precisa el contenido y que afianzan en el niño los procesos cognitivos racionales. Se recomendó capacitación del docente en estrategias didácticas.

Palabras clave: juego educativo; matemáticas; conocimiento. 


\title{
Educational Games Implemented by the Teacher as a Strategy for the Mathematical Knowledge of Children
}

\begin{abstract}
This article represents a significant contribution to the teaching of mathematical discipline. It is titled educational games implemented by the teacher as a strategy for mathematical knowledge. It aimed to describe the educational games implemented by the teacher as a strategy for mathematical knowledge in the children of the EIB "María Clementina de Bonilla" of the municipality of Peña Yaracuy state. Methodologically, it was based on a descriptive study supported by field research. The population was made up of fifteen (15) teachers who constituted the sample in the case of a census sample. A questionnaire with a Likert scale was applied. For the results obtained, descriptive statistics of frequency and percentage represented in tables were used. Among the conclusions, there was a low tendency of teachers to implement educational games as a strategy for mathematical knowledge. Considering little importance to the objectives of the game as an element that requires content and that strengthen the child's rational cognitive processes. Teacher training was recommended in teaching strategies.
\end{abstract}

Keywords: educational games; mathematics; knowledge.

Date Received: 12-07-2018

Date Acceptance: 01-09-2018 


\section{Introducción}

La implementación de estrategias en el estudio del área de la matemática no sólo favorece el pensamiento lógico-matemático de estudiantes, sino también despierta la creatividad, la intuición, la capacidad de análisis y de crítica. En este orden de ideas, las matemáticas han brindado al estudiante un abanico de posibilidades que le permiten asumir actitudes positivas frente al trabajo y perseverar ante soluciones a problemas de la vida cotidiana.

Desde este horizonte, la matemática es avizorada como pilar importante por la función formativa, instrumental y funcional que desempeña en el estudiante al generar la capacidad de construir relaciones, deducción, razonamiento y facilitación de la expresión. En efecto, la enseñanza de la matemática no solo busca la construcción de un lenguaje simbólico, sino construir las significaciones de un razonamiento lógico. Al respecto, cabe destacar el estudio realizado por Flotts, Manzi, Barrios, Saldaña, Mejías y Abarzúa (2016): donde afirman que "la matemática es la vía para..., formar personas capaces de razonar lógicamente y de pensar críticamente..., que dominan ciertos saberes o contenidos propios de esta disciplina, pero que además puedan ser capaz de aplicarlos en la vida cotidiana..." (pág. 26).

Ahora bien, es importante señalar que en el transcurrir de la historia del hombre en lo que respecta al contexto educativo, se ha considerado la enseñanza de la matemática como una asignatura compleja para los niños. Además, en el trabajo de investigación La enseñanza de la matemática, un reto para los maestros del siglo XXI, realizado por López (2014a), explica que:

...existen causas que hacen de la enseñanza y aprendizaje de las matemáticas un proceso tedioso y poco significativo, como por ejemplo: cuando no existen en el aula los medios para este fin; los espacios para la interacción entre docente y estudiantes..., no son los adecuados; los recursos didácticos no son pertinentes o no se cuenta con ellos; las pedagogías y metodologías con las que se desarrolla la clase no están en 
consonancia con los objetivos trazados para la misma... (pág. $57)$.

Por lo tanto, las limitaciones metodológicas, la carencia de una formación científica en los aspectos pedagógicos de esta asignatura, constituyen limitaciones para impartir el saber, sobre todo el de la matemática. De hecho, el aprendizaje de la matemática ha sido considerado por diversos investigadores, como una de las disciplinas que presenta mayor dificultad para los alumnos.

Al respecto, cabe destacar a investigadores dominicanos como Cabrera (2006); Flores y Lapaix (2008); Mejía (2010); Vincent (2010) y González (2011), citados por Lachapell (2017): quienes aseveran "la carencia de enfoques y modelos pedagógicos congruentes con el trabajo del aula, y la débil comprensión de significados matemáticos..., constituyen barreras para el aprendizaje; así como currículos desprovistos de didácticas específicas... del conocimiento matemático" (pág. 331).

Atendiendo a esto, el Sistema Educativo Bolivariano (SEB, 2007), asume, en su Currículo la concepción de las matemáticas caracterizado por:

La implementación de diversas metodologías de trabajo en el contexto de los espacios de aprendizajes; tales como los proyectos, las estaciones de trabajo, las investigaciones colectivas, los talleres, los seminarios, entre otras. Así, actividades como contar, medir, estimar, jugar, explicar y demostrar son importantes para el proceso de orientación y aprendizaje de las matemáticas, aunado al desarrollo de procesos como: representar, sintetizar, generalizar, abstraer, conjeturar y comunicar, entre otros... En este sentido, la educación matemática bajo el enfoque del SEB, contribuye significativamente en desarrollar lo metódico, el pensamiento ordenado y el razonamiento lógico (pág. 21).

Desde esta perspectiva, se debe brindar un ambiente armónico y coherente que permita el logro de competencias y vencer las limitaciones, 
perfeccionando los métodos de enseñanza de la matemática. Al respecto, López (2014b): señala que "las matemáticas, debido a su carácter abstracto, necesitan de ambientes propios, que estimulen en el estudiante el desarrollo del pensamiento matemático y faciliten el proceso de enseñanza y aprendizaje" (pág. 57).

Por tanto, lo expresado por el autor antes citado, permite afirmar que es fundamental la implementación de estrategias pedagógicas más dinámica y constructiva que promuevan en el educando la curiosidad de indagar, de construir su propio aprendizaje desde lo que ya conoce, y de asirse de esos saberes para poder desarrollar su pensamiento lógico matemático sin dificultad. Sin embargo, pareciera ser que las prácticas pedagógicas se estuvieran llevando a cabo en un proceso contrario al que sostiene la concepción constructivista. Al vislumbrarse en la mayoría de los docentes una praxis que no se cimenta sobre la base de lo ya conocido por estudiante, sino más bien, se basa en asignación de ejercicios para la adquisición del dominio algorítmico de las operaciones básicas de la matemática. Conduciendo, esta rutinaria práctica de enseñanza en fobia hacia las matemáticas, fastidio y rechazo.

A la situación descrita, no escapa la Escuela Integral Bolivariana "María Clementina de Bonilla", del municipio Peña del Estado Yaracuy; donde según registro evaluativo evidenciado por el investigador del presente estudio, las evaluaciones del área de matemática en la mayoría de los niños obtuvieron como calificación el literal "C" y muy pocos el literal "B", lo que demuestra que no han adquirido los conocimientos necesarios para expresarse matemáticamente. $Y$ en conversación con el director de dicha institución, expresó que los docentes imparten sus actividades diarias con estrategias convencionales que no estimulan a los niños para superar las dificultades en las áreas académicas, sobre todo en matemática.

Por lo antes expresado se evidencia que el conocimiento de las 
matemáticas es de gran valor formativo; además de necesario, para cualquier tipo de estudio. De allí, que lo expuesto motiva a la idea de favorecer la labor docente, desde la puesta en prácticas de herramientas que proporcionen el estudio de la matemática. En tal sentido, se describen los juegos didácticos como estrategia para la construcción de conocimientos más dinámicos entorno al área de matemática.

En virtud de la situación planteada, cabe preguntarse: ¿Qué juegos educativos implementa frecuentemente el docente? Esta interrogante proporciona la direccionalidad a este estudio y se traducen en el objetivo general de la presente investigación. Describir los juegos educativos implementados por el docente como estrategia para el conocimiento matemático en los niños de la EIB "María Clementina de Bonilla" del municipio Peña estado Yaracuy.

De acuerdo con lo anteriormente señalado, este estudio se justificó y se consideró relevante por cuanto, pretendió describir los juegos educativos implementados por el docente como estrategia para el conocimiento matemático. En tal sentido, contribuyó a mejorar en lo pedagógico, por cuanto planteó el desarrollo del pensamiento lógico matemático mediante juegos educativos como estrategia dirigida a los docentes con la finalidad de convertir su saber en una instrucción educativa más dinámica y divertida para el niño. En el contexto técnico, permitió a los docentes contar con recursos pedagógico que le proporciona un mejor desempeño en su rol de facilitador. $Y$ en el niño disminuir los fracasos escolares.

\section{Marco Teórico Referencial}

Entre los referentes teóricos que constituyen el sintagma gnoseológico, referido al tema de estudio en un contexto hermenéutico el autor de este estudio se apoyó en el trabajo de Quintanilla (2016), titulado:

Estrategias lúdicas dirigidas a la enseñanza de la matemática 
a nivel de educación primaria tuvo como objetivo proponer estrategias lúdicas dirigidas a la enseñanza de la matemática a nivel del primer grado de educación primaria de la Escuela Básica Estadal "Profesora Teresa de Jesús Narza" de la Parroquia Miguel Peña del Municipio Valencia, del Estado Carabobo. Las conclusiones derivadas..., permiten afirmar..., los docentes sí reconocen que la enseñanza de la matemática debe ser orientada de forma práctica y mediante el uso del juego, pero ellos no poseen las estrategias necesarias 0 desconocen cual aplicar. Realidad que sustenta la propuesta donde se pone de manifiesto una serie de estrategias..., para brindar un aprendizaje significativo... (pág. 20).

Este estudio sirvió de base a la presente investigación, por cuanto aportó elementos teóricos que sustentaron los juegos didácticos como una de las herramientas de las que el docente puede utilizar para llevar a cabo su labor educativa en cuanto al saber matemático.

\subsection{Juegos educativos}

De igual manera, se consiguió argumentos que describen los juegos educativos, estrategias didácticas, desarrollo del pensamiento lógico y la teoría Cognoscitiva de Jean Piaget. En este orden de ideas, se consideró como juego educativo a la forma lúdica de organizar la enseñanza. De hecho, Piaget (1976): lo define como "una actividad cuyo fin está en sí misma ejerciendo su adaptación pura en el cual el niño asimila, sin presiones" (pág. 165).

\subsection{Estrategias}

En otro orden de ideas, se fundamentó el estudio en las estrategias didácticas apuntalándola como la vía para la adquisición de objetivos. Cabe parafrasear, lo planteado por Álvarez (2007), citado por Ayrout (2011): quien la concibe como "una metodología de enseñanza de carácter participativa y dialógica..., que genera aprendizajes significativos..." (pág. 12). 


\subsection{Conocimiento matemático}

Por otra parte, se consideró como fundamentación teórica del conocimiento matemático, el pensamiento lógico de acuerdo con lo planteado por Nieves y Torres (2013): quienes afirman es la "capacidad para utilizar los números de manera inductiva y de razonar adecuadamente empleando el pensamiento lógico..., en la generación de ideas, utilización de representaciones de la realidad y la comprensión del entorno que rodea al niño" (pág. 68).

Del mismo modo, el estudio, se orientó en la teoría de Cognoscitiva de Jean Piaget (1956), para este psicólogo según la revista digital para profesionales de la enseñanza, Temas para la Educación (2010):

El juego forma parte de la inteligencia del niño, porque representa la asimilación funcional o reproductiva de la realidad según cada etapa evolutiva del individuo. Las capacidades sensoriomotrices, simbólicas o de razonamiento, como aspectos esenciales del desarrollo del individuo, son las que condicionan el origen y la evolución del juego (pág. 6).

De acuerdo con lo planteado anteriormente, el juego constituye una herramienta para la adquisición del aprendizaje, permite al niño una vivencia significativa, creativa y de desarrollo de sus capacidades. En resumen, la significatividad que representa el juego en la vida del niño favorece la labor docente. Por tanto, el juego puede ser considerado una estrategia para el conocimiento matemático, por cuanto promueve aprendizajes de forma más agradable, sencilla y divertida para los niños.

\section{Metodología}

El estudio tuvo como objetivo principal describir los juegos educativos implementados por el docente como estrategia para el conocimiento matemático. Para ello, el investigador siguió y parafraseo los aportes metodológicos de Hernández, Fernández, y Baptista (2006a): al respecto, 
señalan que "los estudios descriptivos buscan especificar las propiedades importantes de las personas, grupos, comunidades, o cualquier otro fenómeno que sea sometido a análisis" (pág. 60). En este orden de ideas, la investigación fue de carácter descriptivo apoyada en una investigación de campo, por cuanto los datos fueron recogidos directamente en su ambiente natural.

Con relación a la población objeto de estudio, la conformó quince (15) docentes de la EIB "María Clementina de Bonilla" municipio Peña, estado Yaracuy. Por ser una población pequeña, fueron considerados en su totalidad. Al respecto, Hernández, Fernández, y Baptista (2006b): la denomina como "muestra censal" (pág. 243). Para la búsqueda de información, se recurrió a la encuesta tipo cuestionario estructurado en veintiún (21) ítems, siguiendo el método desarrollado por Rensis Likert. EL instrumento fue validado por tres (03) especialista: un (01) metodólogo; (01) en redacción y (01) en el área de matemática. $Y$ para conocer la consistencia interna del instrumento se utilizó la técnica de Alfa de Cronbach, mediante la siguiente fórmula:

$$
\propto=\left[\frac{\mathrm{k}}{\mathrm{k}-1}\right] \quad\left[1-\frac{\sum_{\mathrm{s}}^{2} \mathrm{i}}{\mathrm{s}^{2}}\right]
$$

Donde se obtuvo como resultado:

$$
\begin{gathered}
\propto=\left[\frac{\mathrm{k}}{\mathrm{k}-1}\right] \quad\left[1-\frac{\sum_{\mathrm{s}}^{2} \mathrm{i}}{\mathrm{s}^{2}}\right] \quad \propto=\frac{21}{21-1}\left(1-\frac{3,3}{42}\right) \\
\propto=1,05 . \quad(1-0,07) \\
\propto=1,05 . \quad 0,93 \\
\propto=0,97
\end{gathered}
$$

\section{Resultados}

Para el análisis e interpretación de los resultados, se aplicó la estadística descriptiva de frecuencia y porcentaje de cada ítem. Luego, se representaron en cuadros y se le hizo la correspondiente interpretación 
cuantitativa y cualitativa, como se presentan a continuación:

Tabla 1. Juego Educativo.

\begin{tabular}{|c|c|c|c|c|c|c|c|c|c|c|c|}
\hline \multirow{2}{*}{ ÍTEM } & \multirow{2}{*}{ ENUNCIADO } & \multicolumn{2}{|c|}{ s } & \multicolumn{2}{|c|}{ CS } & \multicolumn{2}{|c|}{ AV } & \multicolumn{2}{|c|}{ CN } & \multicolumn{2}{|c|}{$\mathrm{N}$} \\
\hline & & $f$ & $\%$ & $f$ & $\%$ & f & $\%$ & $f$ & $\%$ & $f$ & $\%$ \\
\hline 1 & $\begin{array}{l}\text { Realiza usted juegos } \\
\text { con sus alumnos } \\
\text { durante el desarrollo } \\
\text { de las clases. }\end{array}$ & 1 & 7 & 3 & 20 & 5 & 33 & 6 & 40 & 0 & 0 \\
\hline 2 & $\begin{array}{l}\text { Les gusta a sus niños } \\
\text { (as) participar en } \\
\text { juegos. }\end{array}$ & 1 & 7 & 6 & 40 & 5 & 33 & 3 & 20 & 0 & 0 \\
\hline 3 & $\begin{array}{c}\text { Toma en cuenta los } \\
\text { conocimientos previos } \\
\text { detectados en los } \\
\text { niños (as) para la } \\
\text { selección de los } \\
\text { juegos educativos. }\end{array}$ & 1 & 7 & 3 & 20 & 5 & 33 & 6 & 40 & 0 & 0 \\
\hline
\end{tabular}

Fuente: El Autor (2016).

Para el ítem 1, referido a si realizan juegos con sus alumnos durante el desarrollo de las clases, se evidenció que el $40 \%$ de los sujetos encuestados expresaron que casi nunca realizan juegos, un 33\% expresó que algunas veces y un $20 \%$ casi siempre.

Con respecto al ítem 2, sobre si a los niños (as) les gusta participar en juegos el $40 \%$ manifestó que casi siempre, un $33 \%$ señaló que algunas veces y el $20 \%$ casi nunca.

En relación con el ítem 3, concerniente a si toman en cuenta los conocimientos previos detectados en los niños (as) para la selección de los juegos educativos un $40 \%$ de los sujetos encuestados expresó que casi nunca consideran los conocimientos previos, un $33 \%$ señaló que algunas veces y un $20 \%$ casi siempre.

Los resultados anteriores mostraron, que hay una baja tendencia de implementación de los juegos educativos por parte de los docentes como 
estrategia para el conocimiento matemático en los niños de la EIB "María Clementina de Bonilla". A pesar de que los juegos son considerados como una herramienta que influye poderosamente en la actuación cognitiva del individuo.

Tabla 2. Estrategia.

\begin{tabular}{|c|c|c|c|c|c|c|c|c|c|c|c|c|}
\hline \multirow{2}{*}{ ÍTEM } & ENUNCIADO & \multicolumn{2}{|c|}{ S } & \multicolumn{3}{|c|}{ CS } & \multicolumn{3}{|c|}{ AV } & \multicolumn{2}{|c|}{ CN } & \multicolumn{2}{|c|}{ N } \\
\cline { 3 - 9 } & $\mathbf{f}$ & $\%$ & $\mathbf{f}$ & $\%$ & $\mathbf{f}$ & $\%$ & $\mathbf{f}$ & $\%$ & $\mathbf{f}$ & $\%$ \\
\hline & $\begin{array}{c}\text { Utiliza usted juegos } \\
\text { o dinámicas para } \\
\text { enseñar a los niños } \\
\text { (as) matemática. }\end{array}$ & & & & 1 & 7 & 3 & 20 & 3 & 20 & 8 & 53 \\
\hline
\end{tabular}

Fuente: El Autor (2016).

En cuanto al ítem 4, relacionado a si el docente utiliza juegos o dinámicas para enseñar a los niños (as) matemática, se evidenció el 53\% de los docentes manifestaron que nunca, $20 \%$ casi nunca y otro $20 \%$ casi siempre. Con dichos resultados, se evidenció que existe una baja tendencia de los docentes en facilitar las clases de matemática de manera divertida. Por consiguiente, se puede afirmar que las clases son monótonas y repetitivas con los mismos métodos y estrategias tradicionales.

Tabla 3. Conocimiento matemático.

\begin{tabular}{|c|c|c|c|c|c|c|c|c|c|c|c|}
\hline \multirow{2}{*}{ ÍTEM } & \multirow{2}{*}{ ENUNCIADO } & \multicolumn{2}{|c|}{$S$} & \multicolumn{2}{|c|}{ CS } & \multicolumn{2}{|c|}{ AV } & \multicolumn{2}{|c|}{ CN } & \multicolumn{2}{|c|}{$\mathbf{N}$} \\
\hline & & $f$ & $\%$ & $f$ & $\%$ & $f$ & $\%$ & $f$ & $\%$ & $f$ & $\%$ \\
\hline 5 & $\begin{array}{l}\text { Utiliza usted juegos } \\
\text { didácticos para el desarrollo } \\
\text { del razonamiento lógico } \\
\text { matemático en las cuatro } \\
\text { operaciones básicas. }\end{array}$ & 0 & 0 & 1 & 7 & 3 & 20 & 5 & 33 & 6 & 40 \\
\hline
\end{tabular}

Fuente: El Autor (2016).

Finalmente, para el ítem 5 donde se preguntó si el docente utiliza juegos educativos para mejorar el razonamiento lógico de las cuatro operaciones básicas, se constató que el $40 \%$ expresó nunca, un 33\% que casi nunca y un $20 \%$ algunas veces. Estos resultados, muestran una baja tendencia de los 
docentes en considerar importante el desarrollo del pensamiento lógico como un proceso básico para la realización de operaciones racionales en matemática.

\section{Conclusiones}

De acuerdo con los objetivos propuestos y la información recabada en el instrumento, surgen las siguientes conclusiones:

- Una baja tendencia de los docentes en implementar los juegos educativos como estrategia para el conocimiento matemático en los niños de la EIB "María Clementina de Bonilla". Considerando poca importancia a los objetivos del juego como elemento que precisa el contenido y que afianzan en el niño los procesos cognitivos racionales.

- Una baja tendencia del docente en realizar actividades lúdicas como estrategia didáctica que permitan o faciliten el desarrollo de destrezas mentales en la realización de operaciones racionales en matemática. Sin embargo, le da importancia a la dramatización como juego simbólico.

- Una baja tendencia de los docentes en utilizar actividades de operaciones lógicas para la construcción de las estructuras internas lógicas-racionales.

\section{Reflexiones Finales}

Los resultados de esta investigación y sus conclusiones, junto a la necesidad perentoria de perfeccionar el desempeño docente, plantearon las siguientes recomendaciones:

- Proponer al personal directivo la capacitación de los docentes mediante talleres relacionados con la implementación de juegos educativos como estrategia para el conocimiento matemático. 
- Más motivación por parte del docente asesor hacia la promoción de los juegos, en los proyectos de aprendizajes ejecutados por el personal de la institución; de manera que los estudiantes puedan desarrollar destrezas mentales en la realización de operaciones racionales en matemática y otras áreas académicas.

- Que los docentes reconozcan los juegos educativos como generador aprendizaje para los estudiantes y de gran valor para el docente, por cuanto le permite desarrollar un conjunto de actividades de manera más motivante para el niño.

- Que las universidades se conviertan en el eje articulador de innovadoras estrategias que permitan el desarrollo del pensamiento lógico matemático.

\section{Referencias}

Álvarez, M. (2007). Estrategias de aprendizaje lúdicas. México: Universidad de Guadalajara.

Ayrout, A. (2011). Efecto de un programa en el uso de estrategias lúdicas dirigido a los docentes de educación primaria. Trabajo especial de maestría. Maracaibo, Venezuela: Universidad Rafael Urdaneta. Recuperado de: http://200.35.84.131/portal/bases/marc/texto/9218-1106704.pdf

Flotts, M., Manzi, J., Barrios, C., Saldaña, V., Mejías, N., \& Abarzúa, A (2016). Aportes para la enseñanza de la matemática. Santiago, Chile: Oficina Regional de Educación para América Latina y el Caribe, OREALC/UNESCO. Recuperado de: http://unesdoc.unesco.org/images/0024/002448/244855S.pdf Hernández, R., Fernández, C., \& Baptista, P. (2006a,b). Metodología de la investigación. México: McGraw-Hill. 
Lachapell, G. (2017) La formación didáctico matemática del docente de la República Dominicana. Transformación, 13(3), 327-337 ISSN: 20772955. Recuperado de: http://scielo.sld.cu/pdf/trf/v13n3/trf04317.pdf

López, G. (2014a,b) La enseñanza de las matemáticas, un reto para los maestros del siglo XXI. Praxis Pedagógica, 14(15), 55-76. Recuperado de:

http://dx.doi.org/10.26620/uniminuto.praxis.14.15.2014.55-76

Nieves, M., \& Torres, A. (2013). Incidencia del desarrollo del pensamiento lógico matemático en la capacidad de resolver problemas matemáticos en los niños y niñas del sexto grado de Educación Básica en la escuela mixta "Federico Malo" de la ciudad de Cuenca durante el año lectivo 2012-2013. Trabajo de grado. Cuenca, Ecuador: Universidad de Politécnica Salesiana. Recuperado de: https://dspace.ups.edu.ec/bitstream/123456789/5576/1/UPS$\underline{\text { CT002787.pdf }}$

Piaget, J. (1976). Génesis de las estructuras lógicas elementales. Buenos Aires, Argentina: Biblioteca Pedagógica Guadalupe.

Quintanilla, N. (2016). Estrategias lúdicas dirigidas a la enseñanza de la matemática a nivel de educación primaria. Tesis de maestría. Bárbula, Carabobo, Venezuela: Universidad Carabobo. Recuperado de: http://mriuc.bc.uc.edu.ve/bitstream/handle/123456789/3882/nquintanill a.pdf?sequence $=1$

SEB (2007). Subsistema de Educación Primaria Bolivariana: Currículo y Orientaciones Metodológicas. Caracas, Venezuela: Sistema Educativo Bolivariano.

Temas para la Educación (2010). La estimulación educativa. Temas para la Educación, (11), 1-10, ISSN: 1989-4023. Recuperado de:

https://www.feandalucia.ccoo.es/docu/p5sd7581.pdf 


\section{Carlos Reinaldo Patiño Espinal}

e-mail: carlosreypati58@gmail.com

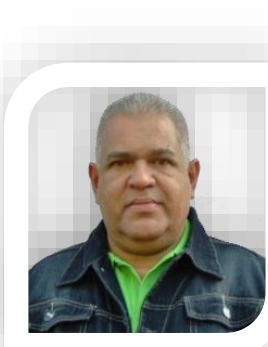

Nacido en Yaritagua, estado Yaracuy, Venezuela.

Licenciado en Educación Integral en la Universidad

Nacional Experimental Simón Rodríguez de

Barquisimeto, estado Lara; Postgrado en Gerencia

Educativa en la Universidad Pedagógica

Experimental Libertador, núcleo académico San

Felipe, Yaracuy; Doctor en Ciencias de la Educación en la Universidad Fermín

Toro, Cabudare, estado Lara. Docente Adscrito a la Secretaria de Educación del estado Yaracuy.

El contenido de este manuscrito se difunde bajo una Licencia de Creative Commons ReconocimientoNoComercial-Compartirlgual 4.0 Internacional 